\title{
OPEN Association between diabetes status and subsequent onset of glaucoma in postmenopausal women
}

\author{
Younhea Jung ${ }^{1}$, Kyungdo Han ${ }^{2}$, Kyoung Ohn ${ }^{1}$, Da Ran Kim ${ }^{1}$ \& Jung II Moon ${ }^{1 凶}$
}

The purpose of this study was to analyze the risk of glaucoma based on diabetes status using a large nationwide longitudinal cohort of postmenopausal women. This study included 1,372,240 postmenopausal women aged $\mathbf{2} \mathbf{4 0}$ years who underwent National Health Screening Program in 2009. Subjects were classified into the following 5 categories based on diabetes status: no diabetes, impaired fasting glucose (IFG), new onset diabetes, diabetes treated with oral hypoglycemic medication, and diabetes treated with insulin. Subjects were followed from 2005 through 2018, and hazard ratios of glaucoma onset were calculated for each group. Subgroup analyses of subjects stratified by age, smoking, drinking, hypertension, and dyslipidemia were performed. During the follow up period, 42,058 subjects developed glaucoma. The adjusted hazard ratio was 1.061 (95\% $\mathrm{Cl}, 1.036-1.086)$ in the IFG group, 1.151 (95\% Cl, 1.086-1.220) in the new onset diabetes group, $1.449(95 \% \mathrm{Cl}, 1.406-1.493)$ in the diabetes treated with oral hypoglycemic medication group, and $1.884(95 \% \mathrm{Cl}, 1.777-1.999)$ in the diabetes treated with insulin group compared to the no diabetes group. The results were consistent in subgroup analyses after stratifying by age, lifestyle factors (smoking and drinking), and comorbidities (hypertension and dyslipidemia). Diabetes status is associated with increased risk of glaucoma development in postmenopausal women.

Diabetes is a global health burden ${ }^{1}$. Although there are various treatments developed to control plasma glucose, the prevalence of diabetes and its macrovascular and microvascular complications is increasing worldwide ${ }^{1,2}$. Glaucoma is also a major burden, which is characterized by irreversible progressive visual field loss corresponding to the loss of retinal ganglion cells, and most patients are asymptomatic until late stage. Therefore, identifying its risk factors for early detection of glaucoma is clinically important ${ }^{3}$.

Diabetes has been suggested to increase the risk of glaucoma by increasing intraocular pressure (IOP) $)^{4-6}$. However, the association between diabetes and IOP was weak in previous studies, and furthermore, the most prevalent type of glaucoma in Korea is normal tension glaucoma ${ }^{7-9}$. In this regard, diabetes has also been suggested to cause microvascular damage and vascular dysregulation of the optic nerve head which increases the susceptibility to glaucomatous damage ${ }^{4-6}$.

Many prior studies report conflicting results on the association between diabetes and glaucoma ${ }^{10-13}$. In addition, there is a relative lack of studies associating glaucoma and impaired fasting glucose (IFG) or effect of treatment for diabetes such as oral hypoglycemic agents or insulin ${ }^{8}$.

Therefore, we conducted a longitudinal study to analyze the risk of glaucoma development according to diabetes status. Specifically, we used a large nationwide cohort of postmenopausal women to determine the risk of glaucoma onset in subjects with no diabetes, IFG, new onset diabetes, diabetes treated with oral hypoglycemic medication, or diabetes treated with insulin.

\section{Methods}

Data source. This study was based on the Korean National Health Insurance Service (KNHIS) database. The KNHIS is a single insurer managed by the Korean government, and provides comprehensive medical care to $97 \%$ of the Korean population ${ }^{14}$. The database contains demographics (anonymized code for each individual, age, sex, socioeconomics, household income, etc.) and medical data (inpatient and outpatient service records,

${ }^{1}$ Department of Ophthalmology, Yeouido St. Mary's Hospital, College of Medicine, The Catholic University of Korea, 10, 63-ro, Yeongdeungpo-gu, Seoul 07345, Republic of Korea. ${ }^{2}$ Department of Statistics and Actuarial Science, Soongsil University, Seoul, Republic of Korea. ${ }^{\circledR}$ email: jimoon@catholic.ac.kr 


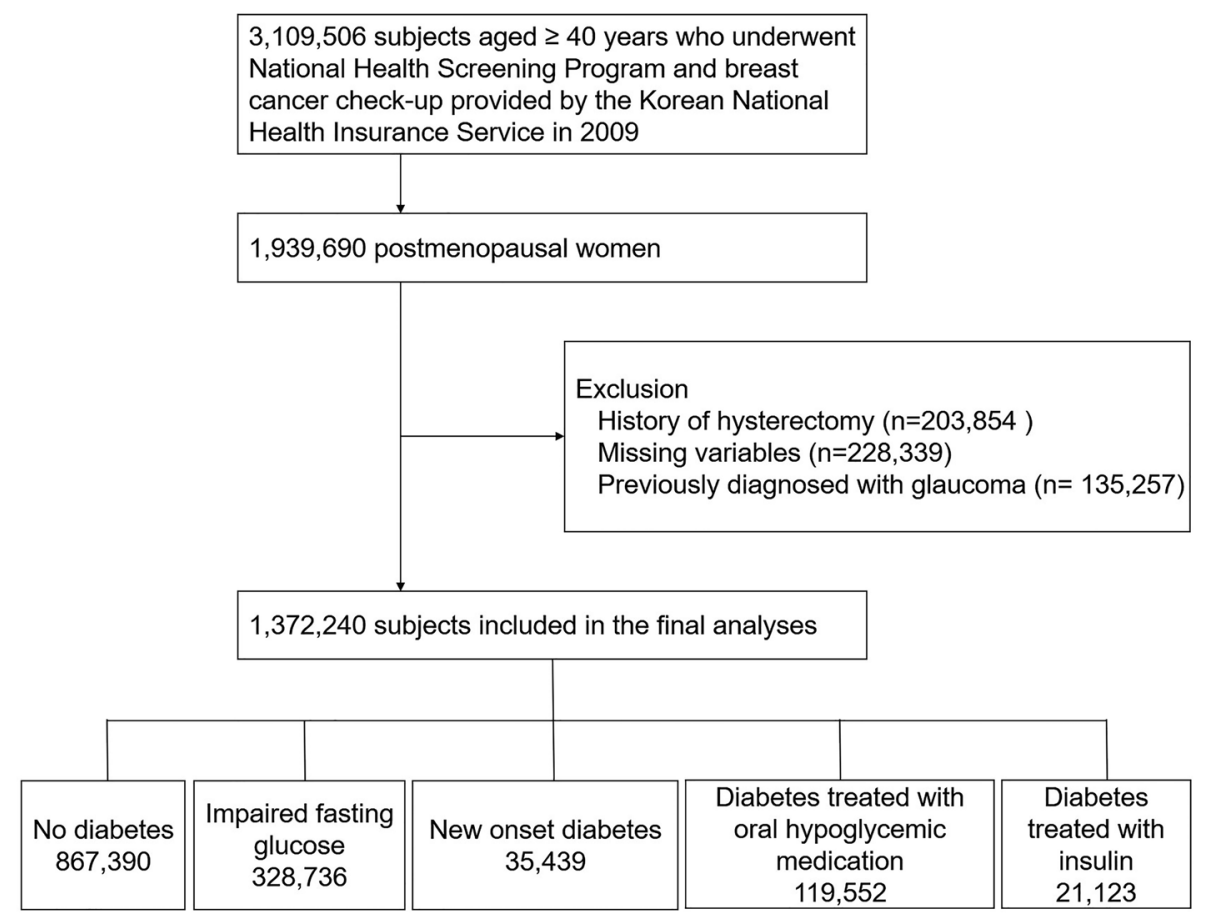

Figure 1. Study subjects.

diagnostic codes classified by the International Classification of Diseases $10^{\text {th }}$ revision [ICD-10], prescriptions, and medical procedures).

The KNHIS also provides a breast cancer check-up along with standardized National Health Screening Program (NHSP) to all women aged $\geq 40$ years insured by the KNHIS. The program includes anthropometric data, a set of laboratory tests, and a self-reported questionnaire with regards to health behaviors. Additionally, it also includes a comprehensive survey encompassing clinical symptoms, weight loss, family history of cancer, and various reproductive factors (age at menarche, age at menopause, history of hormone replacement therapy, parity, breastfeeding, history of oral contraceptive use, etc.).

This study was approved by the Institutional Review Board of the Yeouido St. Mary's Hospital, Seoul, Korea, which waived consent from individual subjects because we used publicly open and anonymized data. Our research adhered to the tenets of the Declaration of Helsinki.

Study population. In the study, 3,109,506 women aged $\geq 40$ years who had undergone NHSP and breast cancer check-up in 2009 (index year) were initially screened (Fig. 1). We used this database, because many covariates were recorded at this check-up. Of these, we selected 1,939,690 subjects who were postmenopause. Subjects with unnatural menopause $(n=203,854)$ due to hysterectomy or those with missing variables $(n=228,339)$ were excluded. Subjects with previously diagnosed glaucoma before the index year $(n=135,257)$ were also excluded. A total of 1,372,240 naturally postmenopausal women without prior history of glaucoma were included in the final analyses and were followed until December 31, 2018. Subjects were censored if they developed glaucoma or died.

Type 2 diabetes was defined using a combination of KNHID claims data and NHSP results. History of diabetes diagnosis was defined as at least one claim per year with E11-E14 (ICD-10 codes) and at least one claim per year with prescription for antidiabetic medication (sulfonylureas, metformin, meglitinides, thiazolidinediones, dipeptidyl peptidase- 4 inhibitors, $\alpha$-glucosidase inhibitors, or insulin ${ }^{15}$. Diabetes status was stratified into 5 categories using the data within one year prior to the index date: (1) normal (no history of diabetes diagnosis and FBS $<100 \mathrm{mg} / \mathrm{dL}$ ), (2) IFG (no history of diabetes diagnosis and $100 \mathrm{mg} / \mathrm{dL} \leq \mathrm{FBS}<126 \mathrm{mg} / \mathrm{dL}$ ), (3) new onset diabetes (no history of diabetes diagnosis and FBS $\geq 126 \mathrm{mg} / \mathrm{dL}$ ), (4) diabetes treated with oral hypoglycemic medication, and (5) diabetes treated with insulin. If a subject was using oral hypoglycemic medication and insulin together, he/she was categorized into the insulin group.

The primary end point was development of glaucoma, which was defined based on ICD-10 code for primary open-angle glaucoma (H401). Those with at least 3 visits for glaucoma were included in the study to enhance the validity of the diagnosis ${ }^{16}$.

Covariates, which were measured during NHSP check-up in 2009, included age, smoking, drinking, exercise, body mass index, parity, breastfeeding, oral contraceptive use, age at menarche, age at menopause, and hormone replacement therapy.

Smoking status was classified into nonsmoker, ex-smoker, or current smoker using the following question: "Have you ever smoked more than five packs of cigarettes in your life?"17. Alcohol drinking was categorized 
as no alcohol, mild alcohol ( $<30 \mathrm{~g}$ per day), or heavy alcohol ( $\geq 30 \mathrm{~g}$ per day). Low income was defined as an annual household income level in the lowest quintile. Regular exercise was defined as moderate-intensity exercise for $\geq 30 \mathrm{~min}, \geq 5$ times a week or vigorous-intensity exercise for $\geq 20 \mathrm{~min}, \geq 3$ times a week. Participants' body mass index (BMI) was calculated as weight $(\mathrm{kg})$ divided by the square of height $\left(\mathrm{m}^{2}\right)$. Systolic and diastolic BP were measured in a seated position after resting more than $5 \mathrm{~min}$. Fasting glucose and total cholesterol were measured with blood samples collected after an overnight fasting.

Comorbidities, including hypertension and dyslipidemia, were based on ICD-10 codes within one year prior to NHSP and NHSP results. Hypertension was defined based on ICD-10 code for hypertension (I10-I13 and I15) with at least one prescription for antihypertensive medication or systolic BP $\geq 140 \mathrm{mmHg}$ or diastolic $\mathrm{BP} \geq 90 \mathrm{mmHg}$. Dyslipidemia was defined as at least one prescription of lipid-lowering medication under ICD10 code for dyslipidemia (E78) or as serum total cholesterol level $\geq 240 \mathrm{mg} / \mathrm{dL}$.

Statistical analysis. All statistical analyses were conducted using SAS (ver 9.4; SAS Institute, Cary, NC, USA) with $P$ values $<0.05$ considered significant.

The baseline characteristics of the study participants were compared in 5 diabetes groups using the Student $t$-test and ANOVA for continuous variables and $\chi^{2}$ test for categorical variables. The incidence rates of endpoint outcome were calculated by dividing the number of events by 1,000 person-years. Cox proportional hazard regression analyses were used to calculate the risk of glaucoma onset according to diabetes status. Hazard ratios (HR) and 95\% confidence intervals (CI) of endpoint outcomes were derived before and after adjusting for potential confounding factors. Subgroup analyses were performed after stratifying by age, smoking, drinking, hypertension, and dyslipidemia. To test the significance of the subgroup effects, interaction terms of diabetes status with age, smoking, drinking, hypertension, and dyslipidemia status were added to the Cox model, respectively, and $P$-values for interaction were reported. Fully-adjusted model included age, income, smoking, drinking, exercise, body mass index, and reproductive factors including parity, breastfeeding, oral contraceptive use, age at menarche, age at menopause, and hormone replacement therapy. Kaplan-Meier curves for incidence probabilities of glaucoma according to diabetes status were generated.

\section{Results}

Baseline characteristics of the study population. A total of 1,372,240 subjects, who underwent natural menopause, were included in the study (Fig. 1). The baseline characteristics of the study population are shown in Table 1. Compared to subjects without diabetes, those with diabetes were older, more likely to be ex- or current smokers, and more obese.

Table 2 shows the risk of glaucoma in postmenopausal women according to diabetes status before and after adjusting for confounding factors. The unadjusted risk of incident glaucoma increased according to diabetes status: in IFG (hazard ratio $[\mathrm{HR}]=1.097,95 \%$ confidence interval $[\mathrm{CI}]=1.072-1.123$ ), in new onset diabetes $(\mathrm{HR}=1.226,95 \% \mathrm{CI}=1.157-1.300)$, diabetes treated with oral hypoglycemic medication $(\mathrm{HR}=1.672,95 \%$ $\mathrm{CI}=1.624-1.722)$, diabetes treated with insulin $(\mathrm{HR}=2.200,95 \% \mathrm{CI}=2.075-2.333)$ in non-adjusted model. After adjusting for confounding factors (age, income, smoking, drinking, exercise, body mass index, and reproductive factors including parity, breastfeeding, oral contraceptive use, age at menarche, age at menopause, and hormone replacement therapy), the adjusted HR of glaucoma was 1.061 (95\% CI $=1.036-1.086), 1.151(95 \%$ $\mathrm{CI}=1.086-1.220), 1.449(95 \% \mathrm{CI}=1.406-1.493)$, and $1.884(95 \% \mathrm{CI}=1.777-1.999)$ in $\mathrm{IFG}$, new onset diabetes, diabetes treated with oral hypoglycemic medication, and diabetes treated with insulin, respectively. The cumulative incidence of glaucoma according to diabetes status is shown in Fig. 2.

The comparison of adjusted HRs (96\% CIs) of glaucoma incidence in subgroups after stratifying by age, smoking, drinking, hypertension, and dyslipidemia is shown in Table 3. The association between diabetes status and subsequent glaucoma was consistent in all subgroup analyses. The risk of incident glaucoma was more prominent in younger age group $(P$ for interaction $<0.001)$ compared to older age group and those without hypertension $(P$ for interaction $<0.001)$ compared to those with hypertension. Figure 3 shows the cumulative incidence of glaucoma according to diabetes status in subgroups.

\section{Discussion}

In this nationwide longitudinal cohort study of Korean adults with natural menopause, type 2 diabetes was associated with an increased risk of glaucoma incidence before and after adjusting for confounding factors. More importantly, diabetes status, stratified into IFG, new onset diabetes, diabetes treated with oral hypoglycemic medication, and diabetes treated with insulin, successively increased the risk of glaucoma. While these findings were consistent in all subgroups, the association was more prominent in younger age group (<65 years) compared to older age group and those without hypertension compared to those with hypertension.

While there are previous studies on the association between diabetes and glaucoma or increased level of intraocular pressure, there is a relative lack of studies associating IFG and glaucoma, and the results are controversial ${ }^{8}$. Choi et al. ${ }^{18}$ reported increased incidence of glaucoma in those with high fasting glucose level. However, high glucose level $\geq 200 \mathrm{mg} / \mathrm{dL}$ was not associated with glaucoma in the Los Angeles Latino Eye Study ${ }^{19}$. Different measurement methods and different ethnicities may have resulted in various results across studies. Our study provides further evidence supporting that IFG also increases the risk of glaucoma development in naturally post-menopausal Korean women. However, the aHR was close to 1, namely 1.061, indicating about $6 \%$ increase in the risk of glaucoma development in patients with IFG compared to normal controls. It is worth noting that IFG was based on a single measurement of fasting blood glucose, and even small increase in HR, we believe, is clinically important. Our results suggest that incidence of glaucoma is proportional to the severity of 


\begin{tabular}{|c|c|c|c|c|c|}
\hline \multirow[b]{3}{*}{$N$} & \multicolumn{5}{|l|}{ Diabetes status } \\
\hline & No diabetes & Impaired fasting glucose & New onset diabetes & $\begin{array}{l}\text { Diabetes treated with oral } \\
\text { hypoglycemic medication }\end{array}$ & Diabetes treated with insulin \\
\hline & 867,390 & 328,736 & 35,439 & 119,552 & 21,123 \\
\hline \multicolumn{6}{|l|}{ Demographics } \\
\hline Age & $60.66 \pm 8.20$ & $61.75 \pm 8.25$ & $62.83 \pm 8.52$ & $64.80 \pm 7.75$ & $65.24 \pm 7.75$ \\
\hline \multicolumn{6}{|l|}{ Smoking } \\
\hline Non & $835,933(96.37)$ & $315,689(96.03)$ & $33,759(95.26)$ & $114,637(95.89)$ & $20,200(95.63)$ \\
\hline Ex & $8890(1.02)$ & $3697(1.12)$ & $390(1.10)$ & $1431(1.20)$ & $276(1.31)$ \\
\hline Current & $22,567(2.60)$ & $9350(2.84)$ & $1290(3.64)$ & $3484(2.91)$ & $647(3.06)$ \\
\hline \multicolumn{6}{|l|}{ Alcohol drinking } \\
\hline No & $757,370(87.32)$ & $281,904(85.75)$ & $30,436(85.88)$ & $109,844(91.88)$ & $19,899(94.21)$ \\
\hline Mild & $106,102(12.23)$ & $44,430(13.52)$ & $4690(13.23)$ & $9264(7.75)$ & $1178(5.58)$ \\
\hline Heavy & $3918(0.45)$ & $2402(0.73)$ & $313(0.88)$ & $444(0.37)$ & $46(0.22)$ \\
\hline Regular Exercise & $161,459(18.61)$ & 59,471 (18.09) & $6053(17.08)$ & $22,648(18.94)$ & $3705(17.54)$ \\
\hline Income (lowest quintile) & $200,051(23.06)$ & $74,451(22.65)$ & $8369(23.62)$ & $25,618(21.43)$ & $4258(20.16)$ \\
\hline \multicolumn{6}{|l|}{ Past medical history } \\
\hline Diabetes mellitus & $0(0)$ & $0(0)$ & $35,439(100)$ & $119,552(100)$ & $21,123(100)$ \\
\hline Hypertension & $336,269(38.77)$ & $167,296(50.89)$ & $21,234(59.92)$ & $85,631(71.63)$ & $16,141(76.41)$ \\
\hline Dyslipidemia & $248,890(28.69)$ & $126,491(38.48)$ & $15,711(44.33)$ & $64,910(54.29)$ & $12,662(59.94)$ \\
\hline Chronic kidney disease & $85,653(9.87)$ & $41,059(12.49)$ & $5373(15.16)$ & $21,144(17.69)$ & $5677(26.88)$ \\
\hline \multicolumn{6}{|l|}{ Laboratory findings } \\
\hline Systolic blood pressure, $\mathrm{mmHg}$ & $123.93 \pm 15.90$ & $127.62 \pm 16.12$ & $130.28 \pm 16.73$ & $129.77 \pm 16.15$ & $129.52 \pm 16.85$ \\
\hline Diastolic blood pressure, $\mathrm{mmHg}$ & $76.21 \pm 10.10$ & $78.04 \pm 10.18$ & $79.16 \pm 10.38$ & $77.87 \pm 10.04$ & $77.02 \pm 10.37$ \\
\hline Fasting blood glucose, $\mathrm{mg} / \mathrm{dL}$ & $88.62 \pm 7.25$ & $107.84 \pm 6.56$ & $147.83 \pm 33.30$ & $133.88 \pm 42.33$ & $148.93 \pm 63.20$ \\
\hline Cholesterol, mg/dL & $206.98 \pm 41.99$ & $214.58 \pm 45.38$ & $218.92 \pm 48.19$ & $199.36 \pm 48.42$ & $195.48 \pm 52.1$ \\
\hline Body mass index, $\mathrm{kg} / \mathrm{cm}^{2}$ & $23.8 \pm 2.99$ & $24.52 \pm 3.15$ & $25.08 \pm 3.36$ & $25.19 \pm 3.35$ & $24.86 \pm 3.42$ \\
\hline \multicolumn{6}{|l|}{ Reproductive factors } \\
\hline Age at menarche, $y$ & $16.41 \pm 1.84$ & $16.48 \pm 1.83$ & $16.56 \pm 1.82$ & $16.58 \pm 1.81$ & $16.59 \pm 1.80$ \\
\hline-11 & $8746(1.01)$ & $3037(0.92)$ & $286(0.81)$ & $917(0.77)$ & $160(0.76)$ \\
\hline $12-15$ & $452,384(52.15)$ & $166,555(50.67)$ & $17,314(48.86)$ & $57,589(48.17)$ & $10,007(47.37)$ \\
\hline $16-$ & $406,260(46.84)$ & $159,144(48.41)$ & $17,839(50.34)$ & $61,046(51.06)$ & $10,956(51.87)$ \\
\hline Age at menopause, $y$ & $49.98 \pm 3.95$ & $50.08 \pm 4.02$ & $50.06 \pm 4.17$ & $50.1 \pm 4.32$ & $49.9 \pm 4.46$ \\
\hline-39 & $14,430(1.66)$ & $5352(1.63)$ & $675(1.90)$ & $2525(2.11)$ & $515(2.44)$ \\
\hline $40-44$ & $48,832(5.63)$ & $18,581(5.65)$ & $2148(6.06)$ & $7465(6.24)$ & $1472(6.97)$ \\
\hline $45-49$ & $239,985(27.67)$ & $88,770(27.00)$ & $9195(25.95)$ & $30,284(25.33)$ & $5466(25.88)$ \\
\hline $50-54$ & $477,880(55.09)$ & $179,398(54.57)$ & $19,271(54.38)$ & 63,299 (52.95) & $10,918(51.69)$ \\
\hline $55-$ & $86,263(9.95)$ & $36,635(11.14)$ & $4150(11.71)$ & 15,979 (13.37) & $2752(13.03)$ \\
\hline Total reproductive years & $33.57 \pm 4.33$ & $33.6 \pm 4.41$ & $33.5 \pm 4.57$ & $33.52 \pm 4.72$ & $33.31 \pm 4.83$ \\
\hline-29 & $116,954(13.48)$ & $45,177(13.74)$ & $5161(14.56)$ & $18,086(15.13)$ & $3458(16.37)$ \\
\hline $30-34$ & $364,967(42.08)$ & $136,751(41.60)$ & $14,731(41.57)$ & $48,688(40.73)$ & $8796(41.64)$ \\
\hline $35-39$ & $333,568(38.46)$ & $124,948(38.01)$ & $13,070(36.88)$ & $43,284(36.21)$ & $7224(34.20)$ \\
\hline $40-$ & $51,901(5.98)$ & $21,860(6.65)$ & $2477(6.99)$ & $9494(7.94)$ & $1645(7.79)$ \\
\hline \multicolumn{6}{|l|}{ Hormone replacement therapy } \\
\hline None & $691,411(79.71)$ & $268,870(81.79)$ & $29,991(84.63)$ & $101,322(84.75)$ & $17,805(84.29)$ \\
\hline$<2$ years & $83,868(9.67)$ & $28,849(8.78)$ & $2501(7.06)$ & $8101(6.78)$ & $1399(6.62)$ \\
\hline $2-5$ years & $35,389(4.08)$ & $11,262(3.43)$ & $961(2.71)$ & $3063(2.56)$ & $543(2.57)$ \\
\hline$\geq 5$ years & $27,363(3.15)$ & $7929(2.41)$ & $692(1.95)$ & $2666(2.23)$ & $501(2.37)$ \\
\hline Unknown & $29,359(3.38)$ & $11,826(3.60)$ & $1294(3.65)$ & $4400(3.68)$ & $875(4.14)$ \\
\hline \multicolumn{6}{|l|}{ Parity } \\
\hline None & $22,117(2.55)$ & $8122(2.47)$ & $817(2.31)$ & $2431(2.03)$ & $410(1.94)$ \\
\hline 1 & $55,875(6.44)$ & $19,628(5.97)$ & $1969(5.56)$ & $5080(4.25)$ & $899(4.26)$ \\
\hline$\geq 2$ & $789,398(91.01)$ & $300,986(91.56)$ & $32,653(92.14)$ & $112,041(93.72)$ & $19,814(93.80)$ \\
\hline \multicolumn{6}{|l|}{ Breastfeeding } \\
\hline None & $60,027(6.92)$ & $21,537(6.55)$ & $2140(6.04)$ & $6013(5.03)$ & 1045 (4.95) \\
\hline$<6$ months & $61,690(7.11)$ & $20,906(6.36)$ & $1830(5.16)$ & $5011(4.19)$ & $833(3.94)$ \\
\hline 6 months-1 year & $157,643(18.17)$ & $56,712(17.25)$ & $5560(15.69)$ & 16,664 (13.94) & $2934(13.89)$ \\
\hline$\geq 1$ year & $588,030(67.79)$ & $229,581(69.84)$ & 25,909 (73.11) & $91,864(76.84)$ & $16,311(77.22)$ \\
\hline \multicolumn{6}{|l|}{ Continued } \\
\hline
\end{tabular}




\begin{tabular}{|l|l|l|l|l|l|l|}
\hline \multirow{2}{*}{} & \multicolumn{5}{|l|}{ Diabetes status } \\
\cline { 2 - 6 } $\boldsymbol{N}$ & No diabetes & Impaired fasting glucose & New onset diabetes & $\begin{array}{l}\text { Diabetes treated with oral } \\
\text { hypoglycemic medication }\end{array}$ & Diabetes treated with insulin \\
\cline { 2 - 7 } & $\mathbf{8 6 7 , 3 9 0}$ & $\mathbf{3 2 8 , 7 3 6}$ & $\mathbf{3 5 , 4 3 9}$ & $\mathbf{1 1 9 , 5 5 2}$ & $\mathbf{2 1 , 1 2 3}$ \\
\hline Oral contraceptive use & $696,490(80.30)$ & $261,485(79.54)$ & $28,383(80.09)$ & $93,811(78.47)$ & $16,506(78.14)$ \\
\hline None & $78,350(9.03)$ & $30,324(9.22)$ & $3051(8.61)$ & $10,811(9.04)$ & $1831(8.67)$ \\
\hline$<1$ year & $50,104(5.78)$ & $20,468(6.23)$ & $2205(6.22)$ & $8828(7.38)$ & $1612(7.63)$ \\
\hline$\geq 1$ year & $42,446(4.89)$ & $16,459(5.01)$ & $1800(5.08)$ & $6102(5.10)$ & $1174(5.56)$ \\
\hline Unknown & &
\end{tabular}

Table 1. Baseline characteristics. All baseline characteristics are statistically significant at 0.001 .

\begin{tabular}{|c|c|c|c|c|c|c|c|}
\hline Diabetes status & $\mathbf{N}$ & Glaucoma & Duration & Incidence rate ${ }^{*}$ & Model 1 & Model 2 & Model 3 \\
\hline No diabetes & 867,390 & 24,264 & $7,098,664.23$ & 3.418 & 1 (Ref.) & 1 (Ref.) & 1 (Ref.) \\
\hline $\begin{array}{l}\text { Impaired fasting } \\
\text { glucose }\end{array}$ & 328,736 & 10,023 & $2,675,849.58$ & 3.745 & $1.097(1.072,1.123)$ & $1.058(1.034,1.083)$ & $\begin{array}{l}1.061 \\
(1.036,1.086)\end{array}$ \\
\hline $\begin{array}{l}\text { New onset } \\
\text { diabetes }\end{array}$ & 35,439 & 1187 & $283,958.39$ & 4.180 & $1.226(1.157,1.300)$ & $1.141(1.076,1.210)$ & $\begin{array}{l}1.151 \\
(1.086,1.220)\end{array}$ \\
\hline $\begin{array}{l}\text { Diabetes treated } \\
\text { with oral } \\
\text { hypoglycemic } \\
\text { medication }\end{array}$ & 119,552 & 5410 & $951,438.58$ & 5.686 & $1.672(1.624,1.722)$ & $1.445(1.402,1.489)$ & $\begin{array}{l}1.449 \\
(1.406,1.493)\end{array}$ \\
\hline $\begin{array}{l}\text { Diabetes treated } \\
\text { with insulin }\end{array}$ & 21,123 & 1174 & $158,184.39$ & 7.421 & $2.2(2.075,2.333)$ & $1.882(1.774,1.996)$ & $\begin{array}{l}1.884 \\
(1.777,1.999)\end{array}$ \\
\hline$P$-value & & & & & $<.0001$ & $<.0001$ & $<.0001$ \\
\hline * & \multicolumn{7}{|l|}{ per 1000} \\
\hline Model 1 & \multicolumn{7}{|c|}{ Non-adjusted } \\
\hline Model 2 & \multicolumn{7}{|c|}{ Adjusted for age, income, smoking, drinking, exercise, body mass index } \\
\hline Model 3 & \multicolumn{7}{|c|}{$\begin{array}{l}\text { Adjusted for age, income, smoking, drinking, exercise, body mass index, and reproductive factors including parity, } \\
\text { breastfeeding, oral contraceptive use, age at menarche, age at menopause, and hormone replacement therapy }\end{array}$} \\
\hline
\end{tabular}

Table 2. Risk of glaucoma according to diabetes status.

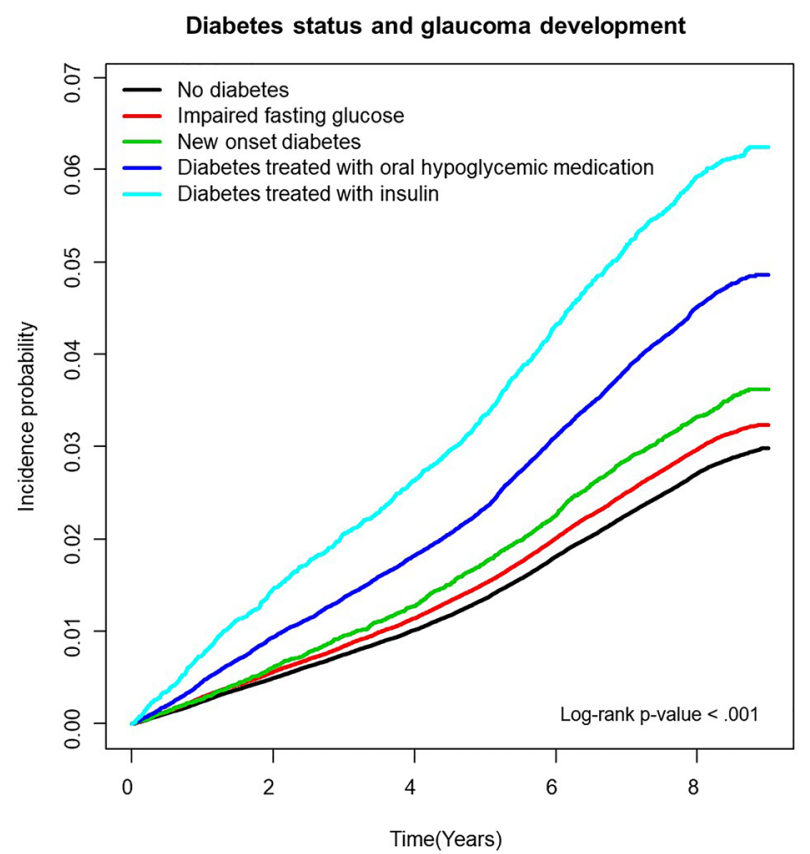

Figure 2. Cumulative incidence of glaucoma according to diabetes status. 


\begin{tabular}{|c|c|c|c|c|c|c|c|}
\hline & Diabetes status & $\mathbf{N}$ & Glaucoma & Duration & Incidence rate ${ }^{*}$ & Model & $P$ for interaction \\
\hline \multicolumn{8}{|l|}{ Age } \\
\hline \multirow[t]{5}{*}{ Age $<65$} & No diabetes & 602,698 & 13,225 & $4,990,467.84$ & 2.650 & 1 (Ref.) & $<.001$ \\
\hline & $\begin{array}{l}\text { Impaired fasting } \\
\text { glucose }\end{array}$ & 213,597 & 5152 & $1,764,649.11$ & 2.920 & $1.076(1.042,1.111)$ & \\
\hline & New onset diabetes & 21,325 & 586 & $174,819.21$ & 3.352 & $1.228(1.130,1.334)$ & \\
\hline & $\begin{array}{l}\text { Diabetes treated } \\
\text { with oral hypogly- } \\
\text { cemic medication }\end{array}$ & 58,817 & 2401 & $481,406.54$ & 4.987 & $1.666(1.594,1.741)$ & \\
\hline & $\begin{array}{l}\text { Diabetes treated } \\
\text { with insulin }\end{array}$ & 9705 & 544 & $76,659.99$ & 7.096 & $\begin{array}{l}2.357 \\
(2.162,2.569)\end{array}$ & \\
\hline \multirow[t]{5}{*}{ Age $\geq 65$} & No diabetes & 264,692 & 11,039 & $2,108,196.38$ & 5.236 & 1 (Ref.) & \\
\hline & $\begin{array}{l}\text { Impaired fasting } \\
\text { glucose }\end{array}$ & 115,139 & 4871 & $911,200.47$ & 5.346 & $1.032(0.998,1.068)$ & \\
\hline & New onset diabetes & 14,114 & 601 & $109,139.19$ & 5.507 & $1.085(0.999,1.178)$ & \\
\hline & $\begin{array}{l}\text { Diabetes treated } \\
\text { with oral hypogly- } \\
\text { cemic medication }\end{array}$ & 60,735 & 3009 & $470,032.05$ & 6.402 & $1.246(1.197,1.298)$ & \\
\hline & $\begin{array}{l}\text { Diabetes treated } \\
\text { with insulin }\end{array}$ & 11,418 & 630 & $81,524.40$ & 7.728 & $1.503(1.387,1.628)$ & \\
\hline \multicolumn{8}{|l|}{ Smoking } \\
\hline \multirow[t]{5}{*}{ No } & No diabetes & 844,823 & 23,700 & $6,917,972.06$ & 3.426 & 1 (Ref.) & 0.541 \\
\hline & $\begin{array}{l}\text { Impaired fasting } \\
\text { glucose }\end{array}$ & 319,386 & 9754 & $2,601,311.44$ & 3.750 & $1.058(1.034,1.084)$ & \\
\hline & New onset diabetes & 34,149 & 1154 & $273,859.12$ & 4.214 & $1.154(1.087,1.224)$ & \\
\hline & $\begin{array}{l}\text { Diabetes treated } \\
\text { with oral hypogly- } \\
\text { cemic medication }\end{array}$ & 116,068 & 5282 & $924,409.81$ & 5.714 & $1.450(1.407,1.495)$ & \\
\hline & $\begin{array}{l}\text { Diabetes treated } \\
\text { with insulin }\end{array}$ & 20,476 & 1140 & $153,556.71$ & 7.424 & $1.876(1.767,1.991)$ & \\
\hline \multirow[t]{5}{*}{ Current } & No diabetes & 22,567 & 564 & $180,692.17$ & 3.121 & 1 (Ref.) & \\
\hline & $\begin{array}{l}\text { Impaired fasting } \\
\text { glucose }\end{array}$ & 9350 & 269 & $74,538.15$ & 3.609 & $1.163(1.004,1.346)$ & \\
\hline & New onset diabetes & 1290 & 33 & $10,099.27$ & 3.268 & $1.051(0.739,1.494)$ & \\
\hline & $\begin{array}{l}\text { Diabetes treated } \\
\text { with oral hypogly- } \\
\text { cemic medication }\end{array}$ & 3484 & 128 & $27,028.77$ & 4.736 & $1.369(1.125,1.666)$ & \\
\hline & $\begin{array}{l}\text { Diabetes treated } \\
\text { with insulin }\end{array}$ & 647 & 34 & 4627.68 & 7.347 & $2.171(1.533,3.075)$ & \\
\hline \multicolumn{8}{|c|}{ Drinking } \\
\hline \multirow[t]{5}{*}{ No } & No diabetes & 757,370 & 21,707 & $6,191,490.16$ & 3.506 & 1 (Ref.) & 0.178 \\
\hline & $\begin{array}{l}\text { Impaired fasting } \\
\text { glucose }\end{array}$ & 281,904 & 8856 & $2,291,470.84$ & 3.865 & $1.063(1.037,1.090)$ & \\
\hline & New onset diabetes & 30,436 & 1063 & $243,485.39$ & 4.366 & $1.169(1.099,1.244)$ & \\
\hline & $\begin{array}{l}\text { Diabetes treated } \\
\text { with oral hypogly- } \\
\text { cemic medication }\end{array}$ & 109,844 & 5005 & $872,936.23$ & 5.734 & $1.443(1.398,1.489)$ & \\
\hline & $\begin{array}{l}\text { Diabetes treated } \\
\text { with insulin }\end{array}$ & 19,899 & 1109 & $148,700.68$ & 7.458 & $1.879(1.768,1.996)$ & \\
\hline \multirow[t]{5}{*}{ Yes } & No diabetes & 110,020 & 2557 & $907,174.07$ & 2.819 & 1 (Ref.) & \\
\hline & $\begin{array}{l}\text { Impaired fasting } \\
\text { glucose }\end{array}$ & 46,832 & 1167 & $384,378.74$ & 3.036 & $1.042(0.972,1.117)$ & \\
\hline & New onset diabetes & 5003 & 124 & $40,473.00$ & 3.064 & $1.015(0.847,1.216)$ & \\
\hline & $\begin{array}{l}\text { Diabetes treated } \\
\text { with oral hypogly- } \\
\text { cemic medication }\end{array}$ & 9708 & 405 & $78,502.35$ & 5.159 & $1.544(1.388,1.718)$ & \\
\hline & $\begin{array}{l}\text { Diabetes treated } \\
\text { with insulin }\end{array}$ & 1224 & 65 & 9483.71 & 6.854 & $2.020(1.578,2.586)$ & \\
\hline \multicolumn{8}{|c|}{ Hypertension } \\
\hline \multirow[t]{4}{*}{ No } & No diabetes & 531,121 & 12,784 & $4,375,071.01$ & 2.922 & 1 (Ref.) & $<.001$ \\
\hline & $\begin{array}{l}\text { Impaired fasting } \\
\text { glucose }\end{array}$ & 161,440 & 4233 & $1,324,433.19$ & 3.196 & $1.075(1.038,1.113)$ & \\
\hline & New onset diabetes & 14,205 & 383 & $115,159.74$ & 3.326 & $1.078(0.973,1.193)$ & \\
\hline & $\begin{array}{l}\text { Diabetes treated } \\
\text { with oral hypogly- } \\
\text { cemic medication }\end{array}$ & 33,921 & 1436 & $274,310.38$ & 5.235 & $1.552(1.469,1.640)$ & \\
\hline
\end{tabular}




\begin{tabular}{|c|c|c|c|c|c|c|c|}
\hline & Diabetes status & $\mathbf{N}$ & Glaucoma & Duration & Incidence rate ${ }^{*}$ & Model & $P$ for interaction \\
\hline & $\begin{array}{l}\text { Diabetes treated } \\
\text { with insulin }\end{array}$ & 4982 & 256 & $38,665.23$ & 6.621 & $1.951(1.724,2.208)$ & \\
\hline \multirow[t]{5}{*}{ Yes } & No diabetes & 336,269 & 11,480 & $2,723,593.22$ & 4.215 & 1 (Ref.) & \\
\hline & $\begin{array}{l}\text { Impaired fasting } \\
\text { glucose }\end{array}$ & 167,296 & 5790 & $1,351,416.39$ & 4.284 & $1.020(0.988,1.052)$ & \\
\hline & New onset diabetes & 21,234 & 804 & $168,798.65$ & 4.763 & $1.139(1.061,1.224)$ & \\
\hline & $\begin{array}{l}\text { Diabetes treated } \\
\text { with oral hypogly- } \\
\text { cemic medication }\end{array}$ & 85,631 & 3974 & $677,128.21$ & 5.869 & $1.346(1.298,1.396)$ & \\
\hline & $\begin{array}{l}\text { Diabetes treated } \\
\text { with insulin }\end{array}$ & 16,141 & 918 & $119,519.16$ & 7.681 & $1.761(1.646,1.884)$ & \\
\hline \multicolumn{8}{|c|}{ Dyslipidemia } \\
\hline \multirow[t]{5}{*}{ No } & No diabetes & 618,500 & 16,446 & $5,063,804.87$ & 3.248 & 1 (Ref.) & 0.076 \\
\hline & $\begin{array}{l}\text { Impaired fasting } \\
\text { glucose }\end{array}$ & 202,245 & 5821 & $1,645,528.60$ & 3.537 & $1.053(1.022,1.085)$ & \\
\hline & New onset diabetes & 19,728 & 643 & $157,626.60$ & 4.079 & $\begin{array}{l}1.163 \\
(1.075,1.259)\end{array}$ & \\
\hline & $\begin{array}{l}\text { Diabetes treated } \\
\text { with oral hypogly- } \\
\text { cemic medication }\end{array}$ & 54,642 & 2436 & $432,544.56$ & 5.632 & $1.464(1.402,1.529)$ & \\
\hline & \begin{tabular}{|l|l|}
$\begin{array}{l}\text { Diabetes treated } \\
\text { with insulin }\end{array}$ \\
\end{tabular} & 8461 & 463 & $62,460.74$ & 7.413 & $1.930(1.759,2.117)$ & \\
\hline \multirow[t]{5}{*}{ Yes } & No diabetes & 248,890 & 7818 & $2,034,859.36$ & 3.842 & 1 (Ref.) & \\
\hline & $\begin{array}{l}\text { Impaired fasting } \\
\text { glucose }\end{array}$ & 126,491 & 4202 & $1,030,320.98$ & 4.078 & $1.044(1.006,1.084)$ & \\
\hline & New onset diabetes & 15,711 & 544 & $126,331.79$ & 4.306 & $1.096(1.005,1.196)$ & \\
\hline & $\begin{array}{l}\text { Diabetes treated } \\
\text { with oral hypogly- } \\
\text { cemic medication }\end{array}$ & 64,910 & 2974 & $518,894.02$ & 5.731 & $1.368(1.310,1.428)$ & \\
\hline & $\begin{array}{l}\text { Diabetes treated } \\
\text { with insulin }\end{array}$ & 12,662 & 711 & $95,723.65$ & 7.428 & $1.751(1.621,1.891)$ & \\
\hline * & per 1000 & & & & & & \\
\hline Model & \multicolumn{7}{|c|}{$\begin{array}{l}\text { Adjusted for age, income, smoking, drinking, exercise, body mass index, and reproductive factors including parity, breast } \\
\text { feeding, oral contraceptive use, age at menarche, age at menopause, and hormone replacement therapy }\end{array}$} \\
\hline
\end{tabular}

Table 3. Risk of glaucoma according to diabetes status stratified by age, smoking, drinking, hypertension, and dyslipidemia.

glycemic burden, and this is the first study to report the association between IFG and glaucoma development in a national scale, longitudinal data.

The mechanisms associating diabetes or IFG to glaucoma are unclear. Hyperglycemia may increase IOP by drawing excess aqueous humor into the anterior chamber ${ }^{20,21}$, or by altering the trabecular meshwork function ${ }^{6}$. However, the association between diabetes and IOP in previous studies was weak ${ }^{7,8}$, and more importantly, in Korea, the most prevalent type of glaucoma is normal tension glaucoma, which suggest that there are other mechanisms beyond increased IOP. Vascular mechanisms also have been proposed to play a role between diabetes and glaucoma even in prediabetic stages ${ }^{22}$. It has been suggested that diabetes causes microvascular damage and vascular dysregulation of the optic nerve head and the retina, thereby increasing the susceptibility to glaucomatous damage ${ }^{19,23}$.

In addition, we found that diabetic patients treated with insulin $(\mathrm{aHR}=1.884)$ were associated with higher risk of glaucoma compared to those treated with oral hypoglycemic agents ( $\mathrm{aHR}=1.449$ ). This association was consistent even in all subgroup analyses before and after adjusting for confounding factors. Previous studies have also reported higher risk for glaucoma in diabetic patients treated with insulin ${ }^{24-26}$. Graw et al. ${ }^{25}$ reported an increased risk of glaucoma (odds ratio of 5.8) in diabetic patients treated with insulin and oral antidiabetics. The Thessaloniki Eye Study also revealed increased risk of glaucoma in those with history of diabetes treated with insulin compared to those treated without insulin ${ }^{24}$. The Baltimore Eyes Study reported increased mean IOP in patients using insulin compared to those without diabetes. In previous studies, diabetes treated with insulin was a self-reported parameter which is subject to potential recall bias, however, in our study, treatment with insulin was based on objective criteria, ICD-10 codes. Although the mechanism is unclear, it could be an effect from insulin itself, or insulin may be a marker for diabetes severity indicating insulin resistance and high glycemic burden ${ }^{24}$. Our findings warrant further research.

While this robust association between diabetes and glaucoma was consistent in all subgroups classified by age, smoking or drinking status, hypertension or dyslipidemia, the association was more prominent in younger age group (<65 years) compared to older age group and those without hypertension compared to those with hypertension. 

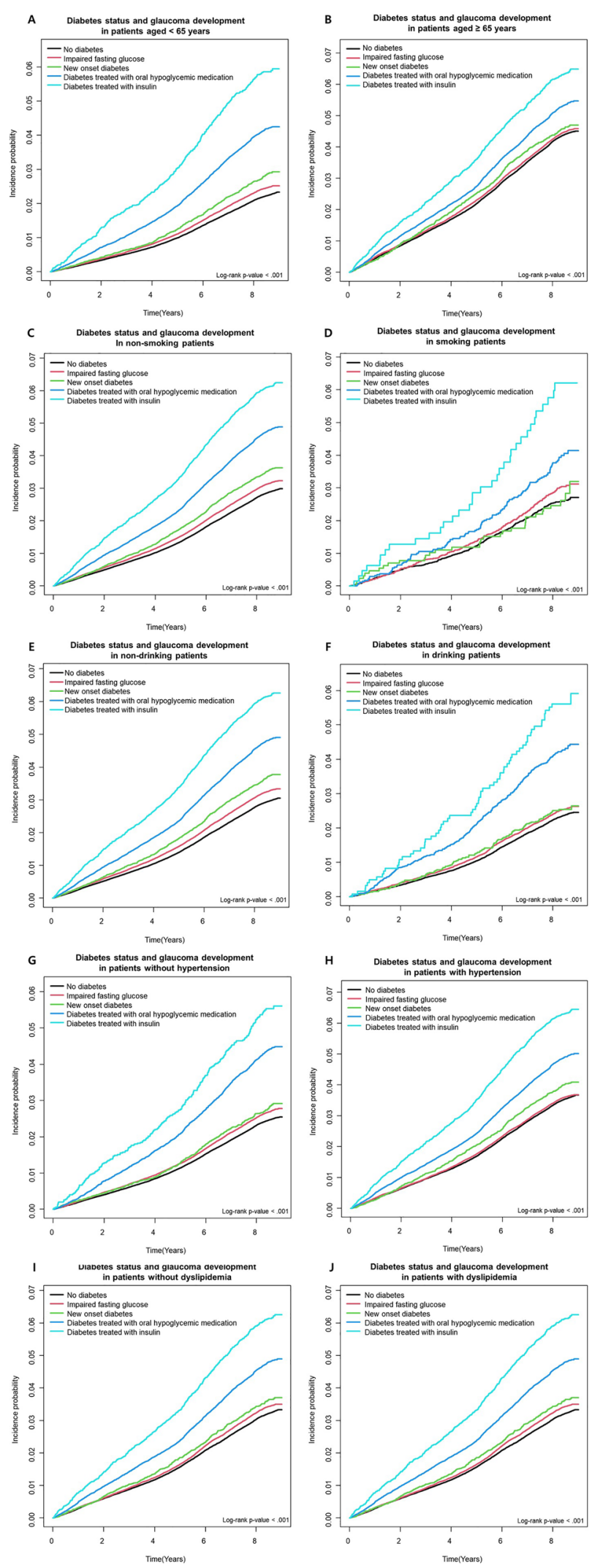

Figure 3. Cumulative incidence of glaucoma according to diabetes status in subgroups after stratifying by age (A and $\mathbf{B})$, smoking ( $\mathbf{C}$ and $\mathbf{D})$, drinking ( $\mathbf{E}$ and $\mathbf{F})$, hypertension $(\mathbf{G}$ and $\mathbf{H})$, and dyslipidemia ( $\mathbf{I}$ and $\mathbf{J})$. 
This study is based on a large population-based epidemiologic cohort with a long follow up period. Our study design using long observation period and eliminating previously diagnosed glaucoma patients enabled us to explore the causality of diabetes status and glaucoma. However, there are also limitations. First, due to the use of claims and health examination database, we did not have access to clinical data regarding severity of diabetes or glaucoma such as HbA1c, IOP measurements, or visual field examinations. Second, our results may partly be affected by selection bias, indicating that diabetic patients may be more likely to receive more frequent eye examinations resulting in overestimation of the relationship between diabetes and glaucoma. In addition, our findings are based on only the Korean population where about $77 \%$ of primary open-angle glaucoma patients have normal IOP ${ }^{9}$ and we only included women. Therefore, our findings cannot be extrapolated to men or other populations where glaucoma results from increased IOP.

In conclusion, in this nationwide population-based longitudinal cohort study, we found that diabetes status is a predictor of glaucoma development in postmenopausal women. Our study suggests that diabetes status can be utilized to select higher-risk groups for glaucoma screening.

\section{Data availability}

Data are available from the Korea National Health Insurance Sharing Service Institutional Data Access Committee (https://nhiss.nhis.or.kr/bd/ay/bdaya001iv.do) for researchers who meet the access criteria.

Received: 30 April 2021; Accepted: 25 August 2021

Published online: 14 September 2021

\section{References}

1. Cho, N. H. et al. IDF diabetes atlas: global estimates of diabetes prevalence for 2017 and projections for 2045. Diabetes Res. Clin. Pract. 138, 271-281 (2018).

2. Unnikrishnan, R., Pradeepa, R., Joshi, S. R. \& Mohan, V. Type 2 diabetes: demystifying the global epidemic. Diabetes 66, $1432-1442$ (2017).

3. Jonas, J. B. et al. Glaucoma. Lancet 390, 2183-2193 (2017).

4. Nakamura, M., Kanamori, A. \& Negi, A. Diabetes mellitus as a risk factor for glaucomatous optic neuropathy. Ophthalmologica 219, 1-10 (2005).

5. Kanamori, A., Nakamura, M., Mukuno, H., Maeda, H. \& Negi, A. Diabetes has an additive effect on neural apoptosis in rat retina with chronically elevated intraocular pressure. Curr. Eye Res. 28, 47-54 (2004).

6. Sato, T. \& Roy, S. Effect of high glucose on fibronectin expression and cell proliferation in trabecular meshwork cells. Invest. Ophthalmol. Vis. Sci. 43, 170-175 (2002).

7. Cohen, E., Kramer, M., Shochat, T., Goldberg, E. \& Krause, I. Relationship between serum glucose levels and intraocular pressure, a population-based cross-sectional study. J. Glaucoma 26, 652-656 (2017).

8. Zhao, D., Cho, J., Kim, M. H., Friedman, D. S. \& Guallar, E. Diabetes, fasting glucose, and the risk of glaucoma: a meta-analysis. Ophthalmology 122, 72-78 (2015).

9. Kim CS, Seong GJ, Lee NH, Song KC, Namil Study Group KGS. Prevalence of primary open-angle glaucoma in central South Korea the Namil study. Ophthalmology 118, 1024-1030 (2011).

10. Investigators, A. The Advanced Glaucoma Intervention Study (AGIS): 12. Baseline risk factors for sustained loss of visual field and visual acuity in patients with advanced glaucoma. Am. J. Ophthalmol. 134, 499-512 (2002).

11. de Voogd, S. et al. Is diabetes mellitus a risk factor for open-angle glaucoma? The Rotterdam Study. Ophthalmology 113, 1827-1831 (2006).

12. Gordon, M. O. et al. The Ocular Hypertension Treatment Study: baseline factors that predict the onset of primary open-angle glaucoma. Arch. Ophthalmol. 120, 714-720 (2002) (discussion 829-730).

13. Mitchell, P., Smith, W., Chey, T. \& Healey, P. R. Open-angle glaucoma and diabetes: the Blue Mountains eye study, Australia. Ophthalmology 104, 712-718 (1997).

14. Lee, J., Lee, J. S., Park, S. H., Shin, S. A. \& Kim, K. Cohort profile: the national health insurance service-national sample cohort (NHIS-NSC), South Korea. Int. J. Epidemiol. 46, e15 (2017).

15. Lee, S. R. et al. Evaluation of the association between diabetic retinopathy and the incidence of atrial fibrillation: a nationwide population-based study. Int. J. Cardiol. 223, 953-957 (2016).

16. Lai, S. W., Lin, C. L. \& Liao, K. F. Glaucoma correlates with increased risk of Parkinson's disease in the elderly: a national-based cohort study in Taiwan. Curr. Med. Res. Opin. 33, 1511-1516 (2017).

17. Lee, S. C. et al. Smoking and the risk of severe acute respiratory syndrome coronavirus 2 (SARS-CoV-2) infection. Nicotine Tob. Res. 2, 19 (2021).

18. Choi, J. A. et al. Fasting plasma glucose level and the risk of open angle glaucoma: Nationwide population-based cohort study in Korea. PLoS One 15, e0239529 (2020).

19. Chopra, V. et al. Type 2 diabetes mellitus and the risk of open-angle glaucoma the Los Angeles Latino Eye Study. Ophthalmology 115, 227-232 e221 (2008).

20. Chun, Y. H. et al. Insulin resistance is associated with intraocular pressure elevation in a non-obese Korean population. PLoS One 10, e112929 (2015).

21. Oh, S. W., Lee, S., Park, C. \& Kim, D. J. Elevated intraocular pressure is associated with insulin resistance and metabolic syndrome. Diabetes Metab. Res. Rev. 21, 434-440 (2005).

22. Wasserman, D. H., Wang, T. J. \& Brown, N. J. The vasculature in prediabetes. Circ. Res. 122, 1135-1150 (2018).

23. Feke, G. T. et al. Effect of brimonidine on retinal vascular autoregulation and short-term visual function in normal tension glaucoma. Am. J. Ophthalmol. 158, 105-112 e101 (2014).

24. Topouzis, F. et al. Risk factors for primary open-angle glaucoma and pseudoexfoliative glaucoma in the Thessaloniki eye study. Am. J. Ophthalmol. 152, 219-228.e211 (2011).

25. Graw, J. et al. The KORA Eye Study: a population-based study on eye diseases in Southern Germany (KORA F4). Invest. Ophthalmol. Vis. Sci. 52, 7778-7786 (2011).

26. Tielsch, J. M., Katz, J., Quigley, H. A., Javitt, J. C. \& Sommer, A. Diabetes, intraocular pressure, and primary open-angle glaucoma in the Baltimore Eye Survey. Ophthalmology 102, 48-53 (1995).

\section{Acknowledgements}

National Health Information Database was provided by the National Health Insurance Service (NHIS) of Korea. The authors would like to thank the National Health Insurance Service for cooperation. 


\section{Author contributions}

Conception and design: Y.J., K.H., and J.M.; data acquisition: Y.J. and K.H.; data analysis and interpretation: Y.J., K.H., K.O., D.K., J.M; statistical analysis: Y.J and K.H.; drafting and finalizing the article: Y.J.; critical revision of the article for important intellectual content: K.H., K.O., D.K., J.M.

\section{Funding}

This work was supported by the National Research Foundation of Korea(NRF) Grant funded by the Korea government(MSIT) (No. 2019R1C1C1011528).

\section{Competing interests}

The authors declare no competing interests.

\section{Additional information}

Correspondence and requests for materials should be addressed to J.I.M.

Reprints and permissions information is available at www.nature.com/reprints.

Publisher's note Springer Nature remains neutral with regard to jurisdictional claims in published maps and institutional affiliations.

(c) (i) Open Access This article is licensed under a Creative Commons Attribution 4.0 International License, which permits use, sharing, adaptation, distribution and reproduction in any medium or format, as long as you give appropriate credit to the original author(s) and the source, provide a link to the Creative Commons licence, and indicate if changes were made. The images or other third party material in this article are included in the article's Creative Commons licence, unless indicated otherwise in a credit line to the material. If material is not included in the article's Creative Commons licence and your intended use is not permitted by statutory regulation or exceeds the permitted use, you will need to obtain permission directly from the copyright holder. To view a copy of this licence, visit http://creativecommons.org/licenses/by/4.0/.

(C) The Author(s) 2021 